\title{
Hedging Long-Term Exposures of a Well-Diversified Portfolio with Short-Term Stock Index Futures Contracts
}

\author{
Yufang Liu, ${ }^{1}$ Wei-Guo Zhang, ${ }^{1}$ Rongda Chen, ${ }^{2,3,4}$ and Junhui Fu ${ }^{2}$ \\ ${ }^{1}$ School of Business Administration, South China University of Technology, Guangzhou 510640, China \\ ${ }^{2}$ School of Finance, Zhejiang University of Finance and Economics, Hangzhou 310018, China \\ ${ }^{3}$ Coordinated Innovation Centre of Wealth Management and Quantitative Investment, Zhejiang University of Finance and Economics, \\ Hangzhou 310018, China \\ ${ }^{4}$ Center for Research of Regulation and Policy of Zhejiang, China
}

Correspondence should be addressed to Junhui Fu; fujh02@gmail.com

Received 20 February 2014; Accepted 3 April 2014; Published 24 April 2014

Academic Editor: Wei Chen

Copyright (C) 2014 Yufang Liu et al. This is an open access article distributed under the Creative Commons Attribution License, which permits unrestricted use, distribution, and reproduction in any medium, provided the original work is properly cited.

\begin{abstract}
It is difficult for passive portfolio strategy to manage the long-term exposure of a well-diversified portfolio because stock index futures contracts have a finite life limited by their maturity. In this paper, we investigate the problem of the rollover hedge strategy for the long-term exposure of a well-diversified portfolio. First, we consider the rollover hedge strategy for the well-diversified portfolio when the portfolio is not adjusted during the period. In order to obtain the optimal solution of the proposed model, the auxiliary models are constructed using the equivalent transformation technique. Moreover, dynamic programming is employed to derive the optimal positions of stock index futures contracts for the long-term exposure of the well-diversified portfolio. In addition, we extend the result to the case of the rollover hedge strategy with transaction costs and derive the optimal number of stock index futures contracts.
\end{abstract}

\section{Introduction}

Stock index futures contracts are often used for the risk management because they have low transaction costs and counterparty risk is small. A key question for stock index futures hedging is how to determine the optimal hedge ratio or the optimal number of stock index futures contracts. The vast majority of the hedging literature has focused on the key question. One of the most widely used methods for calculating the optimal hedge ratio is based on the minimization of the variance of the hedged portfolio. One of the first studies was by Figlewski [1]. He calculated the minimum variance hedge ratio (MVHR) by the ordinary least squares (OLS) method on historical spot and futures returns. On this basis, some studies (e.g., the literature [24]) examined the effectiveness of hedges with duration effect and expiration effects. Lee and Chien [5] investigated the impact of stock market liquidity on the hedging performance of stock index futures by the conditional OLS model with stock market liquidity. Ghosh [6] applied an error correction model (ECM) to capture the lead and lag relationships between stock index and stock index futures and estimated the optimal hedge ratio. He found that ECM incorporated the long-run equilibrium relationship as well as the shortrun dynamics and had significant improvement in estimating the optimal hedge ratio and reducing the risk of risk minimizing portfolio. Eftekhari [7] and Lien and Tse [8] minimized downside risk of the hedged portfolio to obtain the optimal hedge ratio. Chen et al. [9] adopted the meangeneralized semivariance approach to determine the hedge ratio. These studies estimated the optimal hedge ratio under the assumption that the hedge ratio was fixed at the optimum level and was not revised during the hedging period.

The fixed hedge ratio might be inappropriate because the variance and covariance matrix of the spot and futures returns are time-varying over time. Under such circumstances, many dynamic models were proposed to estimate the time-varying hedge ratio. Specifically, the GARCH models are widely used for index futures hedging. The bivariate GARCH models are employed to design the dynamic hedging 
strategy (e.g., the literature [10-17]). The results showed that the hedging strategy based on the bivariate GARCH estimation had better hedging performance than the constant hedging strategy. Sim and Zurbruegg [18] and Wang and Hsu [19] developed error-corrected GARCH models to investigate the dynamic hedging effectiveness in index futures hedging. Yang and Allen [20] and Floros and Vougas [21] examined the hedging effectiveness of multivariate GARCH hedge ratios in Australian and Greek stock index futures market, respectively. Pok et al. [22] and Aragó and Salvador [23] used asymmetric GARCH models to investigate the performance of dynamic hedging strategy.

In addition to the GARCH hedging model, there are several studies using other methods to generate the optimal hedge ratio, such as Markov regime switching approach [24], the exponential weighted moving average (EMWA) method [25], the dynamic hedging algorithm based on a Reverse Order CUSUM-squared testing procedure [26], time-varying copulas methodology $[27,28]$, and autoregressive conditional density (ARCD) models [29].

Although the derivation methods for the time-varying hedge ratio capture the time-varying second moments of financial time series [17], they mainly focused on hedging with one index futures contract. As we know, stock index futures contracts have a finite life span and the most liquid contracts tend to be of short maturity. It is difficult for investors to hedge a long-term exposure with one futures contract. In order to solve the problem, a portfolio of nearby futures contracts, each with different maturities, is used to hedge the long-term exposure. This is the so-called "rollover hedge" strategy.

The "rollover hedge" strategy is mainly applied in hedging the risk of commodities (e.g., the literature [30-34]). But it is strange that the "rollover hedge" strategy is seldom used for stock index futures hedging. The only research of which we are aware that considered the rollover hedge with index futures contracts is by Carchano and Pardo [35]. They devised a rollover hedge strategy with five criteria to manage the long-term exposure of single stock index. However, the issue of hedging the long-term exposure of a well-diversified portfolio was not considered in their studies.

This paper is also closely related to the passive portfolio management. Passive portfolio management is an important strategy in financial investments. The idea of the strategy is to make a well-diversified portfolio with low turnover. Nevertheless, the well-diversified portfolio can do nothing for the systematic risk of the portfolio while it diversifies the unsystematic risk of each security out of the portfolio. In addition, passive portfolio management usually holds the well-diversified portfolio for a long time to reduce the transaction costs. Therefore, it is necessary to discuss the problem of hedging the long-term exposure of a welldiversified portfolio.

The main purpose of this paper is to investigate the rollover hedge for the long-term exposure of the welldiversified portfolio. A new theoretical model is proposed to design the rollover hedge strategy. The rest of the paper is organized as follows. Section 2 presents how to construct the rollover hedging model for the well-diversified portfolio.
Section 3 describes how to solve the proposed model by the equivalent transformation technique and dynamic programming. Section 4 discusses the rollover hedging problem with transaction costs. Finally, we draw a conclusion in Section 5.

\section{The Rollover Hedging Model for the Well- Diversified Portfolio}

In general, passive management funds, such as Barclays Global Investors and State Street Corp., primarily hold a welldiversified portfolio for a long time. To hedge against the risk exposure of the portfolio, they can roll over short-term contracts in stock index futures market. This section lays out the hedge problem of the passive management fund and constructs a new model for hedging the long-term exposure of the well-diversified portfolio without adjustment.

First, we will introduce the rollover hedge strategy with stock index futures. It follows the step below to manage the long-term risk of the portfolio. At the beginning of each period, the passive management fund takes a position in stock index futures contract nearest to expiration. At the end of each period, the former nearby contract is closed out and the subsequent nearby contract is opened. This process is continued over the life of the well-diversified portfolio.

Next, we start to build the new model. The available capital is divided into three separate parts. The first part is invested in the well-diversified portfolio that is not adjusted during the whole investment period. The second part is used for stock index futures contracts. The third part is deposited in the saving account of the bank to deal with the possible liquidity risk.

For the sake of clarity, we use the following notations:

$V_{0}$ : the available investment capital for a passive management fund,

$Q_{j}$ : the number of stock $j$ during the investment period,

$S_{j, t}$ : the price of stock $j$ at time $t$,

$F_{t}$ : the price of stock index futures contract at time $t$,

$Z$ : the unit of stock index futures contract,

$H_{i-1}$ : the number of stock index futures contracts for hedging the long-term exposure,

$\alpha$ : the ratio of the maintenance margin,

$W_{S, t}:$ the capital invested in the well-diversified portfolio at time $t$,

$W_{F, t}$ : the capital invested in stock index futures contracts at time $t$,

$W_{C, t}$ : the saving in the bank at time $t$,

$M_{t}$ : the total amount of $W_{F, t}$ and $W_{C, t}$.

At time $t=t_{0}$, the amount of the well-diversified portfolio is

$$
W_{S, t_{0}}=\sum_{j=1}^{n} Q_{j} S_{j, t_{0}} .
$$


After the well-diversified portfolio is constructed, the total amount of $W_{F, t_{0}}$ and $W_{C, t_{0}}$ is

$$
M_{0}=V_{0}-W_{S, t_{0}}=W_{F, t_{0}}+W_{C, t_{0}}=H_{0} Z F_{t_{0}} \alpha+W_{C, t_{0}},
$$

where $W_{F, t_{0}}$ and $W_{C, t_{0}}$ are the amount of stock index futures contracts and the saving in the bank, respectively.

At time $t=t_{i-1}$, that is, the beginning of the period $i$, the amounts of stock index futures contracts and the saving in the bank are $W_{F, t_{i-1}}=H_{i-1} Z F_{t_{i-1}} \alpha$ and $W_{C, t_{i-1}}$, respectively. Then the total amount of $W_{F, t_{i-1}}$ and $W_{C, t_{i-1}}$ is

$$
M_{i-1}=W_{F, t_{i-1}}+W_{C, t_{i-1}}=H_{i-1} Z F_{t_{i-1}} \alpha+W_{C, t_{i-1}} .
$$

At time $t=t_{i}$, the amounts of stock index futures contracts are expressed by

$$
\begin{aligned}
W_{F, t_{i}} & =H_{i-1} Z F_{t_{i-1}} \alpha+H_{i-1} Z F_{t_{i-1}}-H_{i-1} Z F_{t_{i}} \\
& =H_{i-1} Z\left[F_{t_{i-1}}(1+\alpha)-F_{t_{i}}\right] .
\end{aligned}
$$

At time $t=t_{i}$, that is, the end of the period $i$, the total amount of $W_{F, t_{i}}$ and $W_{C, t_{i}}$ needs to be discussed in two cases because liquidity risk might happen; that is, the balance of the futures account falls below the maintenance margin and the added margin is needed.

If the firm does not face liquidity risk, the saving at time $t=t_{i}$ is

$$
W_{C, t_{i}}=W_{C, t_{i-1}}\left(1+r_{C, i}\right)
$$

where $r_{C, i}$ is the interest rate of current deposits from $t=t_{i-1}$ to $t=t_{i}$.

In the case without liquidity risk, the total amount of $W_{F, t_{i}}$ and $W_{C, t_{i}}$ is

$$
\begin{aligned}
M_{i} & =W_{F, t_{i}}+W_{C, t_{i}} \\
& =H_{i-1} Z\left[F_{t_{i-1}}(1+\alpha)-F_{t_{i}}\right]+W_{C, t_{i-1}}\left(1+r_{C, i}\right) .
\end{aligned}
$$

If the firm receives a margin call and is expected to pay additional margin into the futures account to maintain its position, the amount of the rest capital at time $t=t_{i}$ is different from the case without liquidity risk. The proposed method by $\mathrm{Fu}$ et al. [36] is used to calculate the additional margin.

From $t=t_{i-1}$ to $t=t_{i}$, we assume that there are $N_{i}$ trading days that the balance of the account falls below the deposit required to maintain the position $H_{i-1}$. Let $F_{t_{i-1}+g_{i}(k)}$ be the settlement price of stock index futures contracts on the $k$ th trading day $\left(k=1,2, \ldots, N_{i}, N_{i} \geqq 1\right)$ that the manufacturer receives a margin call, and $g_{i}(k)$ is the number of the trading days between the $k$ th trading day with a margin call and the former with a margin call. In addition, $F_{t_{i-1}+g_{i}(k)}$ should satisfy

$$
\begin{aligned}
F_{t_{i-1}} & <F_{t_{i-1}+g_{i}(1)}<F_{t_{i-1}+g_{i}(2)}<\cdots \\
& <F_{t_{i-1}+g_{i}\left(N_{i}\right)}, t_{i-1}+g_{i}\left(N_{i}\right)<t_{i} .
\end{aligned}
$$

Let $g_{i}(0)=0$; the inequality becomes

$$
\begin{aligned}
F_{t_{i-1}} & =F_{t_{i-1}+g_{i}(0)}<F_{t_{i-1}+g_{i}(1)} \\
& <F_{t_{i-1}+g_{i}(2)}<\cdots<F_{t_{i-1}+g_{i}\left(N_{i}\right)} .
\end{aligned}
$$

At time $t=t_{i-1}+g_{i}(1)$, the balance of the stock index futures account is

$$
H_{i-1} F_{t_{i-1}+g_{i}(0)} \alpha+H_{i-1} F_{t_{i-1}+g_{i}(0)}-H_{i-1} F_{t_{i-1}+g_{i}(1)} .
$$

Then the maintenance margin of stock index futures contracts is $H_{i-1} F_{t_{i-1}+g_{i}(1)} \alpha$. Obviously, the balance of the account is less than the maintenance margin of the position $H_{i-1}$. In order to maintain the position $H_{i-1}$, the amount of the additional margin at time $t_{i-1}+g_{i}(1)$ is

$$
\begin{aligned}
\operatorname{AM}_{t_{i-1}+g_{i}(1)}= & H_{i-1} F_{t_{i-1}+g_{i}(1)} \alpha \\
& -H_{i-1}\left[F_{t_{i-1}+g_{i}(0)} \alpha+F_{t_{i-1}+g_{i}(0)}-F_{t_{i-1}+g_{i}(1)}\right] \\
= & H_{i-1}\left[F_{t_{i-1}+g_{i}(1)}-F_{t_{i-1}+g_{i}(0)}\right](1+\alpha) .
\end{aligned}
$$

After depositing the additional margin $\mathrm{AM}_{t_{i-1}+g_{i}(1)}$, the saving in the bank is

$$
\begin{aligned}
W_{C, t_{i-1}+g_{i}(1)}= & W_{C, t_{i-1}}\left[1+r_{i}(1)\right] \\
& -H_{i-1}\left[F_{t_{i-1}+g_{i}(1)}-F_{t_{i-1}+g_{i}(0)}\right](1+\alpha),
\end{aligned}
$$

where $r_{i}(1)=r \cdot g_{i}(1) / 360$.

At time $t=t_{i-1}+g_{i}(2)$, the balance of the account $H_{i-1}\left[F_{t_{i-1}+g_{i}(1)} \alpha+F_{t_{i-1}+g_{i}(1)}-F_{t_{i-1}+g_{i}(2)}\right]$ is also less than the maintenance margin $H_{i-1} F_{t_{i-1}+g_{i}(2)} \alpha$. The amount of the additional margin should be

$$
\begin{aligned}
\operatorname{AM}_{t_{i-1}+g_{i}(2)}= & H_{i-1} F_{t_{i-1}+g_{i}(2)} \alpha \\
& -H_{i-1}\left[F_{t_{i-1}+g_{i}(1)} \alpha+F_{t_{i-1}+g_{i}(1)}-F_{t_{i-1}+g_{i}(2)}\right] \\
= & H_{i-1}\left[F_{t_{i-1}+g_{i}(2)}-F_{t_{i-1}+g_{i}(1)}\right](1+\alpha) .
\end{aligned}
$$

Then the saving in the bank is

$$
\begin{aligned}
& W_{C, t_{i-1}+g_{i}(2)} \\
&= W_{C, t_{i-1}+g_{i}(1)}\left[1+r_{i}(2)\right] \\
&-H_{i-1}\left[F_{t_{i-1}+g_{i}(2)}-F_{t_{i-1}+g_{i}(1)}\right](1+\alpha) \\
&=\left\{W_{C, t_{i-1}}\left[1+r_{i}(1)\right]-H_{i-1}\left(F_{t_{i-1}+g_{i}(1)}-F_{t_{i-1}+g_{i}(0)}\right)(1+\alpha)\right\} \\
& \times\left[1+r_{i}(2)\right]-H_{i-1}\left[F_{t_{i-1}+g_{i}(2)}-F_{t_{i-1}+g_{i}(1)}\right](1+\alpha) \\
&= W_{C, t_{i-1}} \prod_{m=1}^{2}\left[1+r_{i}(m)\right]-H_{i-1}\left(F_{t_{i-1}+g_{i}(1)}-F_{t_{i-1}+g_{i}(0)}\right) \\
& \times(1+\alpha)\left[1+r_{i}(2)\right] \\
&-H_{i-1}\left[F_{t_{i-1}+g_{i}(2)}-F_{t_{i-1}+g_{i}(1)}\right](1+\alpha),
\end{aligned}
$$

where $r_{i}(2)=r \cdot g_{i}(2) / 360$. 
By analogy, the amounts of the additional margin and the saving at time $t_{i-1}+g_{i}\left(N_{i}\right)$ should be

$$
\begin{aligned}
\mathrm{AM}_{t_{i-1}+g_{i}\left(N_{i}\right)}= & H_{i-1}\left[F_{t_{i-1}+g_{i}\left(N_{i}\right)}-F_{t_{i-1}+g_{i}\left(N_{i}-1\right)}\right](1+\alpha), \\
W_{C, t_{i-1}+g_{i}\left(N_{i}\right)}= & W_{C, t_{i-1}+g_{i}\left(N_{i}-1\right)}\left[1+r_{i}\left(N_{i}\right)\right] \\
& -H_{i-1}\left[F_{t_{i-1}+g_{i}\left(N_{i}\right)}-F_{t_{i-1}+g_{i}\left(N_{i}-1\right)}\right](1+\alpha) \\
= & \left\{W_{C, t_{i-1}+g_{i}\left(N_{i}-2\right)}\left[1+r_{i}\left(N_{i}-1\right)\right]\right. \\
& \left.-H_{i-1}\left[F_{t_{i-1}+g_{i}\left(N_{i}-1\right)}-F_{t_{i-1}+g_{i}\left(N_{i}-2\right)}\right](1+\alpha)\right\} \\
& \times\left[1+r_{i}\left(N_{i}\right)\right] \\
& -H_{i-1}\left[F_{t_{i-1}+g_{i}\left(N_{i}\right)}-F_{t_{i-1}+g_{i}\left(N_{i}-1\right)}\right](1+\alpha) \\
= & W_{C, t_{i-1}+g_{i}\left(N_{i}-2\right)} \prod_{m=N_{i}-1}^{N_{i}}\left[1+r_{i}(j)\right] \\
& -H_{i-1}\left[F_{t_{i-1}+g_{i}\left(N_{i}-1\right)}-F_{t_{i-1}+g_{i}\left(N_{i}-2\right)}\right] \\
& \times(1+\alpha)\left[1+r_{i}\left(N_{i}\right)\right] \\
& -H_{i-1}\left[F_{t_{i-1}+g_{i}\left(N_{i}\right)}-F_{t_{i-1}+g_{i}\left(N_{i}-1\right)}\right](1+\alpha) \\
= & W_{C, t_{i-1}} \prod_{m=1}^{N_{i}}\left[1+r_{i}(j)\right]-H_{i-1} A_{i-1}, \\
\vdots & \\
& \\
& \\
& \\
&
\end{aligned}
$$

where

$$
\begin{aligned}
A_{i-1}= & \sum_{k=1}^{N_{i}}\left\{\left[F_{t_{i-1}+g_{i}(k)}-F_{t_{i-1}+g_{i}(k-1)}\right](1+\alpha) \prod_{m=k+1}^{N_{i}}\left[1+r_{i}(m)\right]\right\} \\
& +\left[F_{t_{i-1}+g_{i}\left(N_{i}\right)}-F_{t_{i-1}+g_{i}\left(N_{i}-1\right)}\right](1+\alpha) .
\end{aligned}
$$

At time $t=t_{i}$, the saving in the bank is given by

$$
\begin{aligned}
W_{C, t_{i}}= & W_{C, t_{i-1}+g_{i}\left(N_{i}\right)}\left[1+r_{i}\left(N_{i}+k_{i}\right)\right] \\
= & \left\{W_{C, t_{i-1}} \prod_{m=1}^{N_{i}}\left[1+r_{i}(j)\right]-H_{i-1} A_{i-1}\right\} \\
& \times\left[1+r_{i}\left(N_{i}+k_{i}\right)\right],
\end{aligned}
$$

where $r_{i}\left(N_{i}+k_{i}\right)=r \cdot g_{i}\left(N_{i}+k_{i}\right) / 360$ and $g_{i}\left(N_{i}+k_{i}\right)$ is the number of the trading days from time $t_{i-1}+g_{i}\left(N_{i}\right)$ to time $t_{i}$.

At the end of period $i$, the total amount of $W_{F, t_{i}}$ and $W_{C, t_{i}}$ with liquidity risk is

$$
\begin{aligned}
M_{i}= & W_{F, t_{i}}+W_{C, t_{i}} \\
= & H_{i-1} Z\left[F_{t_{i-1}}(1+\alpha)-F_{t_{i}}\right] \\
& +\left[W_{C, t_{i-1}} \prod_{m=1}^{N_{i}}\left[1+r_{i}(j)\right]-H_{i-1} A_{i-1}\right] \\
& \times\left[1+r_{i}\left(N_{i}+k_{i}\right)\right] \\
= & H_{i-1} Z\left[F_{t_{i-1}}(1+\alpha)-F_{t_{i}}\right] \\
& +W_{C, t_{i-1}}\left[1+r_{i}\left(N_{i}+k_{i}\right)\right] \prod_{m=1}^{N_{i}}\left[1+r_{i}(j)\right] \\
& -H_{i-1} A_{i-1}\left[1+r_{i}\left(N_{i}+k_{i}\right)\right] \\
= & H_{i-1}\left\{Z\left[F_{t_{i-1}}(1+\alpha)-F_{t_{i}}\right]-A_{i-1}\left[1+r_{i}\left(N_{i}+k_{i}\right)\right]\right\} \\
& +W_{C, t_{i-1}}\left[1+r_{i}\left(N_{i}+k_{i}\right)\right] \prod_{m=1}^{N_{i}}\left[1+r_{i}(j)\right] .
\end{aligned}
$$

Let

$$
\begin{gathered}
e_{F, i-1}= \begin{cases}Z\left[F_{t_{i-1}}(1+\alpha)-F_{t_{i}}\right], & \text { without liquidity risk, } \\
Z\left[F_{t_{i-1}}(1+\alpha)-F_{t_{i}}\right]-A_{i-1}\left[1+r_{i}\left(N_{i}+k_{i}\right)\right], & \text { with liquidity risk, }\end{cases} \\
e_{C, i-1}= \begin{cases}1+r_{C, i}, & \text { without liquidity risk, } \\
{\left[1+r_{i}\left(N_{i}+k_{i}\right)\right] \prod_{m=1}^{N_{i}}\left[1+r_{i}(j)\right],} & \text { with liquidity risk. }\end{cases}
\end{gathered}
$$

According to (6) and (17), the total amount of $W_{F, t_{i}}$ and $W_{C, t_{i}}$ at the end of the period $i$ can be given by

$$
M_{i}=H_{i-1} e_{F, i-1}+W_{C, t_{i-1}} e_{C, i-1} .
$$

Substituting (3) into (19), the total amount of $W_{F, t_{i}}$ and $W_{C, t_{i}}$ is expressed by

$$
\begin{aligned}
M_{i} & =H_{i-1} e_{F, i-1}+W_{C, t_{i-1}} e_{C, i-1} \\
& =H_{i-1} e_{F, i-1}+\left(M_{i-1}-H_{i-1} Z F_{t_{i-1}} \alpha\right) e_{C, i-1} \\
& =M_{i-1} e_{C, i-1}+H_{i-1}\left(e_{F, i-1}-Z F_{t_{i-1}} \alpha e_{C, i-1}\right) .
\end{aligned}
$$


Let $P_{i-1}=e_{F, i}-Z F_{t_{i-1}} \alpha e_{C, i-1}$. Then (20) can be simplified

$$
M_{i}=M_{i-1} e_{C, i-1}+H_{i-1} P_{i-1} .
$$

During the whole period, the terminal profit of the investment is

$$
\Delta R=\left(M_{T}+W_{S, t_{T}}\right)-\left(M_{0}+W_{S, t_{0}}\right),
$$

where $W_{S, t_{T}}=\sum_{j=1}^{n} Q_{j} S_{j, t_{T}}$.

The variance of terminal profit of the investment is

$$
\begin{aligned}
\operatorname{Var}(\Delta R)= & \operatorname{Var}\left(M_{T}+W_{S, t_{T}}\right) \\
= & \operatorname{Var}\left(M_{T}\right)+2 \operatorname{Cov}\left(M_{T}, W_{S, t_{T}}\right)+\operatorname{Var}\left(W_{S, t_{T}}\right) \\
= & E\left(M_{T}^{2}\right)-E^{2}\left(M_{T}\right)+2 E\left(M_{T} W_{S, t_{T}}\right) \\
& -2 E\left(W_{S, t_{T}}\right) E\left(M_{T}\right)+\operatorname{Var}\left(W_{S, t_{T}}\right) .
\end{aligned}
$$

The firm chooses the number of index futures contracts by minimizing the variance of terminal profit of the investment:

$$
\min \operatorname{Var}(\Delta R)=\operatorname{Var}\left(M_{T}+W_{S, t_{T}}\right) .
$$

Combining (24) with (21), we obtain the following model for hedging the long-term exposure of the well-diversified portfolio:

$$
\begin{array}{ll}
\min & \operatorname{Var}\left(M_{T}+W_{S, t_{T}}\right) \\
\text { s.t. } & M_{i}=M_{i-1} e_{C, i-1}+H_{i-1} P_{i-1} .
\end{array}
$$

\section{The Analytical Solution for the Proposed Model}

This section derives the minimum-risk hedge positions for the rollover hedge strategies. The method by $\mathrm{Li}$ and $\mathrm{Ng}$ [37] is employed. Model (25) is nonseparable in the sense of dynamic programming. It is necessary for the optimal solutions of the proposed model to transform model (25) into a separable model.

By equivalent transformation of the objective function, model (25) is rewritten as

$$
\begin{array}{ll}
\max & -\operatorname{Var}\left(M_{T}+W_{S, t_{T}}\right) \\
\text { s.t. } & M_{i}=M_{i-1} e_{C, i-1}+H_{i-1} P_{i-1} .
\end{array}
$$

Let

$$
\begin{aligned}
U\{ & \left.E\left(M_{T}^{2}\right), E\left(M_{T}\right), E\left(M_{T} W_{S, t_{T}}\right)\right\} \\
= & -\operatorname{Var}\left(M_{T}+W_{S, t_{T}}\right) \\
= & -E\left(M_{T}^{2}\right)+E^{2}\left(M_{T}\right)-2 E\left(M_{T} W_{S, t_{T}}\right) \\
& +2 E\left(W_{S, t_{T}}\right) E\left(M_{T}\right)-\operatorname{Var}\left(W_{S, t_{T}}\right) .
\end{aligned}
$$

Then model (26) is expressed by

$$
\begin{array}{ll}
\max & U\left\{E\left(M_{T}^{2}\right), E\left(M_{T}\right), E\left(M_{T} W_{S, t_{T}}\right)\right\} \\
\text { s.t. } & M_{i}=M_{i-1} e_{C, i-1}+H_{i-1} P_{i-1} .
\end{array}
$$

In order to obtain the analytical solution for model (28), we can construct the following auxiliary model:

$$
\begin{aligned}
(\mathrm{AU}) \max & E\left\{-M_{T}^{2}+\lambda M_{T}-2 M_{T} W_{S, t_{T}}\right\} \\
\text { s.t. } & M_{i}=M_{i-1} e_{C, i-1}+H_{i-1} P_{i-1} .
\end{aligned}
$$

Under certain conditions, the auxiliary model (29) is equivalent to model (28). In other words, the set of the optimal solutions of the auxiliary model (29) is also the one of model (28). In the following, we will give the condition for equivalent transformation of the two models. Define $Z_{M}$ and $Z_{A}$ to be the set of models (28) and (29), respectively.

Theorem 1. For any $\pi^{*} \in Z_{M}, \pi^{*} \in Z_{A}$.

Proof. By contradiction, assume that $\pi^{*} \notin Z_{A}$. Then, there exists a $\pi$ such that

$$
\begin{gathered}
\left.\left(-1,2 E\left(M_{T}\right)+2 E\left(W_{S, t_{T}}\right),-2\right)\left[\begin{array}{c}
E\left(M_{T}^{2}\right) \\
E\left(M_{T}\right) \\
E\left(M_{T} W_{S, t_{T}}\right)
\end{array}\right]\right|_{\pi} \\
>\left.\left(-1,2 E\left(M_{T}\right)+2 E\left(W_{S, t_{T}}\right),-2\right)\left[\begin{array}{c}
E\left(M_{T}^{2}\right) \\
E\left(M_{T}\right) \\
E\left(M_{T} W_{S, t_{T}}\right)
\end{array}\right]\right|_{\pi^{*}} .
\end{gathered}
$$

It is obvious that $U\left\{E\left(M_{T}^{2}\right), E\left(M_{T}\right), E\left(M_{T} W_{S, t_{T}}\right)\right\}$ is a convex function of $E\left(M_{T}^{2}\right), E\left(M_{T}\right)$, and $E\left(M_{T} W_{S, t_{T}}\right)$. So the following property is satisfied:

$$
\begin{aligned}
U\{ & \left.E\left(M_{T}^{2}\right), E\left(M_{T}\right), E\left(M_{T} W_{S, t_{T}}\right)\right\}\left.\right|_{\pi} \\
\geq & \left.U\left\{E\left(M_{T}^{2}\right), E\left(M_{T}\right), E\left(M_{T} W_{S, t_{T}}\right)\right\}\right|_{\pi^{*}} \\
& +\left(-1,2 E\left(M_{T}\right)+2 E\left(W_{S, t_{T}}\right),-2\right) \\
& \times\left\{\left.\left[\begin{array}{c}
E\left(M_{T}^{2}\right) \\
E\left(M_{T}\right) \\
\sum_{j=1}^{n} Q_{j} S_{j, t_{T}}
\end{array}\right]\right|_{\pi}-\left.\left[\begin{array}{c}
E\left(M_{T}^{2}\right) \\
E\left(M_{T}\right) \\
\sum_{j=1}^{n} Q_{j} S_{j, t_{T}}
\end{array}\right]\right|_{\pi^{*}}\right\} .
\end{aligned}
$$

According to the analysis above, we have

$$
\begin{aligned}
& \left.U\left\{E\left(M_{T}^{2}\right), E\left(M_{T}\right), E\left(M_{T} W_{S, t_{T}}\right)\right\}\right|_{\pi} \\
& \quad \geq\left. U\left\{E\left(M_{T}^{2}\right), E\left(M_{T}\right), E\left(M_{T} W_{S, t_{T}}\right)\right\}\right|_{\pi^{*}} .
\end{aligned}
$$


It shows that the above inequation contradicts the assumption of $\pi^{*} \notin Z_{A}$. Thus, the proof of Theorem 1 is ended.

Theorem 2. Assume $\pi^{*} \in Z_{A}$. A necessary condition for $\pi^{*} \in$ $Z_{M}$ is

$$
\lambda=2 E\left(M_{T}\right)+2 E\left(W_{S, t_{T}}\right) .
$$

Proof. Obviously, the solution set of the auxiliary model (29) can be parameterized by $\lambda$. In other words, $E\left(M_{T}^{2}\right), E\left(M_{T}\right)$, and $E\left(M_{T} W_{S, t_{T}}\right)$ can be expressed in terms of $E\left(M_{T}^{2}(\lambda)\right)$, $E\left(M_{T}(\lambda)\right)$, and $E\left(W_{S, t_{T}} M_{T}(\lambda)\right)$. Then we can obtain

$$
\begin{aligned}
\max _{\lambda} U & \left\{E\left(M_{T}^{2}(\lambda)\right), E\left(M_{T}(\lambda)\right), E\left(W_{S, t_{T}} M_{T}(\lambda)\right)\right\} \\
= & \max _{\lambda}-E\left(M_{T}^{2}(\lambda)\right)+E^{2}\left(M_{T}(\lambda)\right)-2 E\left(W_{S, t_{T}} M_{T}(\lambda)\right) \\
& +2 E\left(W_{S, t_{T}}\right) E\left(M_{T}(\lambda)\right)-\operatorname{Var}\left(W_{S, t_{T}}\right) .
\end{aligned}
$$

A first-order necessary condition for optimal $\lambda^{*}$ is

$$
\begin{aligned}
& -\frac{\partial E\left(M_{T}^{2}\left(\lambda^{*}\right)\right)}{\partial \lambda}+2\left[E\left(M_{T}\right)+E\left(W_{S, t_{T}}\right)\right] \frac{\partial E\left(M_{T}\left(\lambda^{*}\right)\right)}{\partial \lambda} \\
& -2 \frac{\partial E\left(W_{S, t_{T}} M_{T}\left(\lambda^{*}\right)\right)}{\partial \lambda}=0 .
\end{aligned}
$$

According to Reid and Citron [38], when $\pi^{*} \in Z_{A}$, we get the following property:

$$
\begin{gathered}
-\frac{\partial E\left(M_{T}^{2}\left(\lambda^{*}\right)\right)}{\partial \lambda}+\lambda \frac{\partial E\left(M_{T}\left(\lambda^{*}\right)\right)}{\partial \lambda} \\
-2 \frac{\partial E\left(W_{S, t_{T}} M_{T}\left(\lambda^{*}\right)\right)}{\partial \lambda}=0 .
\end{gathered}
$$

Since the vector $\left[-1,2 E\left(M_{T}\right)+2 E\left(W_{S, t_{T}}\right),-2\right]$ is proportional to $[-1, \lambda,-2]$, the condition necessary for $\pi^{*} \in Z_{M}$ is $\lambda=2 E\left(M_{T}\right)+2 E\left(W_{S, t_{T}}\right)$ under the assumption of $\pi^{*} \epsilon$ $Z_{A}$.

It should become clear that the optimal solution of model (28) is generated by solving the auxiliary model (29). The optimal solution of the auxiliary model (29) can be derived analytically using dynamic programming.

At first, we consider the case of period $T-1$.

Substituting (21) into $E\left\{-M_{T}^{2}+\lambda M_{T}-2 M_{T} W_{S, t_{T}}\right\}$, the optimization problem is given as follows:

$$
\begin{aligned}
\max J_{T-1}\left(H_{T-1} \mid M_{T-1}\right) \\
=\max E\left\{-M_{T}^{2}+\lambda M_{T}-2 M_{T} W_{S, t_{T}}\right\} \\
=\max E\left\{-\left(M_{T-1} e_{C, T-1}+P_{T-1} H_{T-1}\right)^{2}\right. \\
\left.+\left(\lambda-2 W_{S, t_{T}}\right)\left(M_{T-1} e_{C, T-1}+P_{T-1} H_{T-1}\right)\right\}
\end{aligned}
$$

$$
\begin{aligned}
=\max \{ & -H_{T-1}^{2} E\left(P_{T-1}^{2}\right) \\
+ & {\left[-2 M_{T-1} E\left(e_{C, T-1} P_{T-1}\right)+\lambda E\left(P_{T-1}\right)\right.} \\
& \left.\quad-2 E\left(W_{S, t_{T}} P_{T-1}\right)\right] H_{T-1} \\
- & M_{T-1}^{2} E\left(e_{C, T-1}^{2}\right)+\lambda M_{T-1} E\left(e_{C, T-1}\right) \\
- & \left.2 M_{T-1} E\left(W_{S, t_{T}} e_{C, T-1}\right)\right\} .
\end{aligned}
$$

Solving the first-order necessary optimality condition of (37), we get

$$
H_{T-1}^{*}=\frac{(\lambda / 2) E\left(P_{T-1}\right)-M_{T-1} E\left(e_{C, T-1} P_{T-1}\right)-E\left(W_{S, t_{T}} P_{T-1}\right)}{E\left(P_{T-1}^{2}\right)} .
$$

Substituting (38) into (37), the optimal $J_{T-1}\left(H_{T-1} \mid M_{T-1}\right)$ is

$$
\begin{aligned}
& J_{T-1}^{*}\left(M_{T-1}\right) \\
& \quad=-\omega_{T-1} M_{T-1}^{2}+\left(\lambda \delta_{T-1}+K\right) M_{T-1}+\frac{\lambda^{2}}{4} \beta_{T-1}+\gamma,
\end{aligned}
$$

where

$$
\begin{gathered}
\omega_{T-1}=E\left(e_{C, T-1}^{2}\right)-\frac{E^{2}\left(e_{C, T-1} P_{T-1}\right)}{E\left(P_{T-1}^{2}\right)}, \\
\delta_{T-1}=E\left(e_{C, T-1}\right)-\frac{E\left(P_{T-1}\right) E\left(e_{C, T-1} P_{T-1}\right)}{E\left(P_{T-1}^{2}\right)}, \\
K=2\left[\frac{E\left(W_{S, t_{T}} P_{T-1}\right) E\left(e_{C, T-1} P_{T-1}\right)}{E^{2}\left(P_{T-1}^{2}\right)}-E\left(W_{S, t_{T}} e_{C, T-1}\right)\right], \\
\gamma=\frac{E^{2}\left(P_{T-1}\right)}{E\left(P_{T-1}^{2}\right)}, \\
\left.E\left(W_{S, t_{T}} P_{T-1}\right)-\lambda E\left(P_{T-1}\right)\right] E\left(W_{S, t_{T}} P_{T-1}\right) \\
E\left(P_{T-1}^{2}\right)
\end{gathered}
$$

Next, we discuss the case of period $T-1$. Substituting (21) into (39), (39) can be rewritten as

$$
\begin{aligned}
& J_{T-1}^{*}\left(M_{T-1}\right) \\
&= \max J_{T-2}\left(H_{T-2} \mid M_{T-2}\right) \\
&= \max E\left\{-\omega_{T-1} M_{T-1}^{2}+\left(\lambda \delta_{T-1}+K\right) M_{T-1}+\frac{\lambda^{2}}{4} \beta_{T-1}+\gamma\right\} \\
&=\max E\left\{-\omega_{T-1}\left(M_{T-2} e_{C, T-2}+P_{T-2} H_{T-2}\right)^{2}\right. \\
&+\left(\lambda \delta_{T-1}+K\right)\left(M_{T-2} e_{C, T-2}+P_{T-2} H_{T-2}\right) \\
&\left.+\frac{\lambda^{2}}{4} \beta_{T-1}+\gamma\right\}
\end{aligned}
$$




$$
\begin{aligned}
=\max \{ & -\omega_{T-1} H_{T-2}^{2} E\left(P_{T-2}^{2}\right) \\
+ & {\left[\left(\lambda \delta_{T-1}+K\right) E\left(P_{T-2}\right)\right.} \\
& \left.\quad-2 \omega_{T-1} M_{T-2} E\left(e_{C, T-2} P_{T-2}\right)\right] H_{T-2} \\
- & \omega_{T-1} M_{T-2}^{2} E\left(e_{C, T-2}^{2}\right) \\
+ & \left(\lambda \delta_{T-1}+K\right) M_{T-2} E\left(e_{C, T-2}\right) \\
+ & \left.+\frac{\lambda^{2}}{4} \beta_{T-1}+\gamma\right\} .
\end{aligned}
$$

The optimal solution of (41) is given by

$$
H_{T-2}^{*}=\frac{\left(\left(\lambda \delta_{T-1}+K\right) / 2 \omega_{T-1}\right) E\left(P_{T-2}\right)-M_{T-2} E\left(e_{C, T-2} P_{T-2}\right)}{E\left(P_{T-2}^{2}\right)} .
$$

Then (41) can be rewritten as

$$
\begin{aligned}
J_{T-2}^{*}\left(M_{T-2}\right)= & -\omega_{T-1} \omega_{T-2} M_{T-2}^{2}+\left(\lambda \delta_{T-1}+K\right) \delta_{T-2} M_{T-2} \\
& +\frac{\left(\lambda \delta_{T-1}+K\right)^{2}}{4 \omega_{T-1}} \beta_{T-2}+\frac{\lambda^{2}}{4} \beta_{T-1}+\gamma
\end{aligned}
$$

where

$$
\begin{gathered}
\omega_{T-2}=E\left(e_{C, T-2}^{2}\right)-\frac{E^{2}\left(e_{C, T-2} P_{T-2}\right)}{E\left(P_{T-2}^{2}\right)}, \\
\delta_{T-2}=E\left(e_{C, T-2}\right)-\frac{E\left(P_{T-2}\right) E\left(e_{C, T-2} P_{T-2}\right)}{E\left(P_{T-2}^{2}\right)}, \\
\beta_{T-2}=\frac{E^{2}\left(P_{T-2}\right)}{E\left(P_{T-2}^{2}\right)} .
\end{gathered}
$$

Similarly, we can derive the optimal $H_{i}$ and $J_{i}\left(M_{i}\right)(0 \leq$ $i \leq T-3)$

$$
\begin{aligned}
H_{i}^{*}= & \left(\frac{\left(\lambda \delta_{T-1}+K\right)}{2 \omega_{T-1}} \prod_{k=i+1}^{T-2} \frac{\delta_{k}}{\omega_{k}} E\left(P_{i}\right)\right. \\
& \left.-M_{i} E\left(e_{C, i} P_{i}\right)\right) \\
& \times\left(E\left(P_{i}^{2}\right)\right)^{-1}, \\
J_{i}^{*}\left(M_{i}\right)= & -M_{i}^{2} \prod_{k=i}^{T-1} \omega_{k}+\left(\lambda \delta_{T-1}+K\right) M_{i} \prod_{k=i}^{T-2} \delta_{k} \\
& +\frac{\left(\lambda \delta_{T-1}+K\right)^{2}}{4 \omega_{T-1}}\left[\sum_{k=i}^{T-3}\left(\beta_{k} \prod_{s=k+1}^{T-2} \frac{\delta_{s}^{2}}{\omega_{s}}\right)\right] \\
& +\frac{\left(\lambda \delta_{T-1}+K\right)^{2}}{4 \omega_{T-1}} \beta_{T-2}+\frac{\lambda^{2}}{4} \beta_{T-1}+\gamma,
\end{aligned}
$$

where

$$
\begin{aligned}
& \omega_{k}=E\left(e_{C, k}^{2}\right)-\frac{E^{2}\left(e_{C, k} P_{k}\right)}{E\left(P_{k}^{2}\right)}, \\
& \delta_{k}=E\left(e_{C, k}\right)-\frac{E\left(P_{k}\right) E\left(e_{C, k} P_{k}\right)}{E\left(P_{k}^{2}\right)}, \\
& \beta_{k}=\frac{E^{2}\left(P_{k}\right)}{E\left(P_{k}^{2}\right)} .
\end{aligned}
$$

With the optimal solution of the auxiliary model (29), we can solve model (28).

Substituting (38) and (45) into (21), the total amount of $W_{F, T}$ and $W_{C, T}$ can be expressed by the following analytical form:

$$
M_{i}= \begin{cases}M_{0}\left[e_{C, 0}-\frac{P_{0} E\left(e_{C, 0} P_{0}\right)}{E\left(P_{0}^{2}\right)}\right]+\frac{\left(\left(\lambda \delta_{T-1}+K\right) / 2 \omega_{T-1}\right) \prod_{k=1}^{T-1}\left(\delta_{k} / 2 \omega_{k}\right) E\left(P_{0}\right)}{E\left(P_{0}^{2}\right)}, & i=1 ; \\ M_{0} \prod_{k=0}^{i-1}\left[e_{C, k}-\frac{P_{k} E\left(e_{C, k} P_{k}\right)}{E\left(P_{k}^{2}\right)}\right]+\frac{\left(\lambda \delta_{T-1}+K\right)}{2 \omega_{T-1}} & \\ \quad \times \sum_{g=0}^{i-2}\left\{\frac{P_{g} E\left(P_{g}\right)}{E\left(P_{g}^{2}\right)} \prod_{k=g+1}^{T-2} \frac{\delta_{k}}{\omega_{k}} \prod_{k=g+1}^{i-1}\left[e_{C, k}-\frac{P_{k} E\left(e_{C, k} P_{k}\right)}{E\left(P_{k}^{2}\right)}\right]\right\} & 2 \leq i \leq T-2 ; \\ +\frac{\left(\lambda \delta_{T-1}+K\right)}{2 \omega_{T-1}} \prod_{k=i}^{T-2} \frac{\delta_{k}}{\omega_{k}} \frac{P_{i-1} E\left(P_{i-1}\right)}{E\left(P_{i-1}^{2}\right)}, & i=T-1 ; \\ M_{T-2}\left[e_{C, T-2}-\frac{P_{T-2} E\left(e_{C, T-2} P_{T-2}\right)}{E\left(P_{T-2}^{2}\right)}\right]+\frac{\lambda \delta_{T-1}+K}{2 \omega_{T-1}} \frac{P_{T-2} E\left(P_{T-2}\right)}{E\left(P_{T-2}^{2}\right)}, & i=T . \\ M_{T-1}\left[e_{C, T-1}-\frac{P_{T-1} E\left(e_{C, T-1} P_{T-1}\right)}{E\left(P_{T-1}^{2}\right)}\right]+\frac{(\lambda / 2) P_{T-1} E\left(P_{T-1}\right)-P_{T-1} E\left(W_{S, t_{T}} P_{T-1}\right)}{E\left(P_{T-1}^{2}\right)} & i=1,\end{cases}
$$


Combining (48) with the necessary condition $\lambda=2 E\left(M_{T}\right)+$ $2 E\left(W_{S, t_{T}}\right)$, we have

$$
\begin{aligned}
\lambda= & 2 E\left(M_{T}\right)+2 E\left(W_{S, t_{T}}\right) \\
= & 2 M_{0} \prod_{k=0}^{T-1} \delta_{k}+\frac{\left(\lambda \delta_{T-1}+K\right)}{\omega_{T-1}} \sum_{g=0}^{T-4}\left\{\beta_{g} \prod_{k=g+1}^{T-2} \frac{\delta_{k}}{\omega_{k}} \prod_{k=g+1}^{T-1} \omega_{k}\right\} \\
& +\frac{\left(\lambda \delta_{T-1}+K\right)}{\omega_{T-1}} \frac{\delta_{T-2}}{\omega_{T-2}} \beta_{T-3} \prod_{k=T-2}^{T-1} \delta_{k} \\
& +\frac{\lambda \delta_{T-1}+K}{\omega_{T-1}} \beta_{T-2} \delta_{T-1}+\lambda \beta_{T-1} \\
& -\frac{2 E\left(P_{T-1}\right) E\left(W_{S, t_{T}} P_{T-1}\right)}{E\left(P_{T-1}^{2}\right)}+2 E\left(W_{S, t_{T}}\right) .
\end{aligned}
$$

By solving the above equation, we can obtain

$$
\lambda=\frac{2 \omega_{T-1}\left[M_{0} \prod_{k=0}^{T-1} \delta_{k}+E\left(W_{S, t_{T}}\right)\right]+K \eta}{\omega_{T-1}\left(1-\beta_{T-1}\right)-\delta_{T-1} \eta},
$$

where

$$
\begin{aligned}
\eta= & \sum_{g=0}^{T-4}\left\{\beta_{g} \prod_{k=g+1}^{T-2} \frac{\delta_{k}}{\omega_{k}} \prod_{k=g+1}^{T-1} \omega_{k}\right\} \\
& +\frac{\delta_{T-2} \beta_{T-3}}{\omega_{T-2}} \prod_{k=T-2}^{T-1} \delta_{k}+\beta_{T-2} \delta_{T-1} .
\end{aligned}
$$

(49) Then the optimal solution of the proposed model is

$$
H_{i}^{*}= \begin{cases}\frac{\left(\left(\lambda \delta_{T-1}+K\right) / 2 \omega_{T-1}\right) \prod_{k=i+1}^{T-2}\left(\delta_{k} / \omega_{k}\right) E\left(P_{i}\right)-M_{i} E\left(e_{C, i} P_{i}\right)}{E\left(P_{i}^{2}\right)}, & 0 \leq i \leq T-3, \\ \frac{\left.\left(\lambda \delta_{T-1}+K\right) / 2 \omega_{T-1}\right) E\left(P_{T-2}\right)-M_{T-2} E\left(e_{C, T-2} P_{T-2}\right)}{E\left(P_{T-2}^{2}\right)}, & i=T-2, \\ \frac{(\lambda / 2) E\left(P_{T-1}\right)-M_{T-1} E\left(e_{C, T-1} P_{T-1}\right)-E\left(W_{S, t_{T}} P_{T-1}\right)}{E\left(P_{T-1}^{2}\right)}, & i=T-1,\end{cases}
$$

where $M_{i}$ and $\lambda$ satisfy (48) and (50), respectively.

\section{The Rollover Hedging Model with Transaction Costs}

The change of stock index futures contracts is essential in the rollover hedge strategy and causes lots of transaction costs, which has a great impact on the hedging performance. In this section, we will focus on the rollover hedging model with transaction costs. Suppose that $\theta$ is the transaction cost rate of stock index futures contracts.

At time $t=t_{0}$, the capital invested in stock index futures contracts is the sum of the maintenance margin and the transaction cost of stock index futures contracts, that is,

$$
W_{F, t_{0}}=H_{0} Z F_{t_{0}} \alpha+H_{0} Z F_{t_{0}} \theta=H_{0} Z F_{t_{0}}(\alpha+\theta) .
$$

So the total amount of $W_{F, t_{0}}$ and $W_{C, t_{0}}$ is

$Y_{0}=V_{0}-W_{S, t_{0}}=W_{F, t_{0}}+W_{C, t_{0}}=H_{0} Z F_{t_{0}}(\alpha+\theta)+W_{C, t_{0}}$.
At time $t=t_{i-1}$, the capital invested in stock index futures contracts is

$$
W_{F, t_{i-1}}=H_{i-1} Z F_{t_{i-1}} \alpha+H_{i-1} Z F_{t_{i-1}} \theta=H_{i-1} Z F_{t_{i-1}}(\alpha+\theta) .
$$

Then the total amount of $W_{F, t_{i-1}}$ and $W_{C, t_{i-1}}$ is

$$
Y_{i-1}=W_{F, t_{i-1}}+W_{C, t_{i-1}}=H_{i-1} Z F_{t_{i-1}}(\alpha+\theta)+W_{C, t_{i-1}}
$$

Similarly, the total amount of $W_{F, t_{i}}$ and $W_{C, t_{i}}$ also needs to be discussed in two cases.

If the firm does not face liquidity risk, the amounts of stock index futures contracts and the saving at time $t=t_{i}$ are

$$
\begin{gathered}
W_{F, t_{i}}=H_{i-1} Z F_{t_{i-1}} \alpha+H_{i-1} Z F_{t_{i-1}}-H_{i-1} Z F_{t_{i}}-H_{i-1} Z F_{t_{i}} \theta \\
=H_{i-1} Z\left[F_{t_{i-1}}(1+\alpha)-F_{t_{i}}(1+\theta)\right] \\
W_{C, t_{i}}=W_{C, t_{i-1}}\left(1+r_{C, i}\right) .
\end{gathered}
$$


Then the total amount of $W_{F, t_{i}}$ and $W_{C, t_{i}}$ is

$$
\begin{aligned}
Y_{i}= & W_{F, t_{i}}+W_{C, t_{i}} \\
= & H_{i-1} Z\left[F_{t_{i-1}}(1+\alpha)-F_{t_{i}}(1+\theta)\right] \\
& +W_{C, t_{i-1}}\left(1+r_{C, i}\right) .
\end{aligned}
$$

If there is liquidity risk during period $i$, the saving at time $t=t_{i}$ is given by

$$
\begin{aligned}
W_{C, t_{i}}= & W_{C, t_{i-1}+g_{i}\left(N_{i}\right)}\left[1+r_{i}\left(N_{i}+k_{i}\right)\right] \\
= & \left\{W_{C, t_{i-1}} \prod_{m=1}^{N_{i}}\left[1+r_{i}(j)\right]-H_{i-1} A_{i-1}\right\} \\
& \times\left[1+r_{i}\left(N_{i}+k_{i}\right)\right]
\end{aligned}
$$

$$
e_{K, i-1}= \begin{cases}Z\left[F_{t_{i-1}}(1+\alpha)-F_{t_{i}}(1+\theta)\right], & \text { without liquidity risk, } \\ Z\left[F_{t_{i-1}}(1+\alpha)-F_{t_{i}}(1+\theta)\right]-A_{i-1}\left[1+r_{i}\left(N_{i}+k_{i}\right)\right], & \text { with liquidity risk. }\end{cases}
$$

and the total amount of $W_{F, t_{i}}$ and $W_{C, t_{i}}$ is

$$
\begin{aligned}
Y_{i}= & W_{C, t_{i}}+W_{F, t_{i}} \\
= & \left\{W_{C, t_{i-1}} \prod_{m=1}^{N_{i}}\left[1+r_{i}(j)\right]-H_{i-1} A_{i-1}\right\} \\
& \times\left[1+r_{i}\left(N_{i}+k_{i}\right)\right] \\
& +H_{i-1} Z\left[F_{t_{i-1}}(1+\alpha)-F_{t_{i}}(1+\theta)\right] \\
= & H_{i-1}\left\{Z\left[F_{t_{i-1}}(1+\alpha)-F_{t_{i}}(1+\theta)\right]\right. \\
& \left.\quad-A_{i-1}\left[1+r_{i}\left(N_{i}+k_{i}\right)\right]\right\} \\
& +W_{C, t_{i-1}}\left[1+r_{i}\left(N_{i}+k_{i}\right)\right] \prod_{m=1}^{N_{i}}\left[1+r_{i}(j)\right] .
\end{aligned}
$$

Let
Then the total amount of $W_{F, t_{i}}$ and $W_{C, t_{i}}$ can be rewritten as

$$
Y_{i}=H_{i-1} e_{K, i-1}+W_{C, t_{i-1}} e_{C, i-1} .
$$

Substituting (56) into (60), the total amount of $W_{F, t_{i}}$ and $W_{C, t_{i}}$ is expressed by

$$
\begin{aligned}
Y_{i} & =H_{i-1} e_{K, i-1}+W_{C, t_{i-1}} e_{C, i-1} \\
& =H_{i-1} e_{K, i-1}+\left[Y_{i-1}-H_{i-1} Z F_{t_{i-1}}(\alpha+\theta)\right] e_{C, i-1} \\
& =H_{i-1}\left[e_{K, i-1}-Z F_{t_{i-1}}(\alpha+\theta) e_{C, i-1}\right]+Y_{i-1} e_{C, i-1} .
\end{aligned}
$$

Let $L_{i-1}=e_{K, i-1}-Z F_{t_{i-1}}(\alpha+\theta) e_{C, i-1}$. Then the total amount of $W_{F, t_{i}}$ and $W_{C, t_{i}}$ can be simplified as

$$
Y_{i}=H_{i-1} L_{i-1}+Y_{i-1} e_{C, i-1} \text {. }
$$

During the whole period, the terminal profit of the investment is

$$
\Delta V=V_{T}-V_{0}=Y_{T}+W_{S, t_{T}}-\left(Y_{0}+W_{S, t_{0}}\right),
$$

where $V_{T}$ is the terminal wealth at the end of the investment period.

The optimal number of stock index futures contracts is determined by minimizing the variance of terminal profit of the investment:

$$
\min \operatorname{Var}(\Delta V)=\operatorname{Var}\left(Y_{T}+W_{S, t_{T}}\right)
$$

Combining (66) with (64), we can propose the rollover hedging model with transaction costs

$$
\begin{array}{ll}
\min & \operatorname{Var}\left(Y_{T}+W_{S, t_{T}}\right) \\
\text { s.t. } & Y_{i}=H_{i-1} L_{i-1}+Y_{i-1} e_{C, i-1} .
\end{array}
$$

Model (67) is also nonseparable in the sense of dynamic programming and needs to be transformed into a separable model. 
Let

$$
\begin{aligned}
U\{ & \left.E\left(Y_{T}^{2}\right), E\left(Y_{T}\right), E\left(Y_{T} W_{S, t_{T}}\right)\right\} \\
= & -\operatorname{Var}\left(Y_{T}+W_{S, t_{T}}\right) \\
= & -E\left(Y_{T}^{2}\right)+E^{2}\left(Y_{T}\right)-2 E\left(Y_{T} W_{S, t_{T}}\right) \\
& +2 E\left(W_{S, t_{T}}\right) E\left(Y_{T}\right)-\operatorname{Var}\left(W_{S, t_{T}}\right) .
\end{aligned}
$$

Then model (67) is expressed by

$$
\begin{array}{ll}
\max & U\left\{E\left(Y_{T}^{2}\right), E\left(Y_{T}\right), E\left(Y_{T} W_{S, t_{T}}\right)\right\} \\
\text { s.t. } & Y_{i}=H_{i-1} L_{i-1}+Y_{i-1} e_{C, i-1} .
\end{array}
$$

In order to obtain the analytical solution for model (69), we use the following auxiliary model:

$$
(\mathrm{AU} 2): \begin{array}{ll}
\max & E\left\{-Y_{T}^{2}+\lambda Y_{T}-2 Y_{T} W_{S, t_{T}}\right\} \\
\text { s.t. } & Y_{i}=H_{i-1} L_{i-1}+Y_{i-1} e_{C, i-1}
\end{array}
$$

Define $Z_{\mathrm{MU}}$ and $Z_{\mathrm{AU}}$ to be the set of models (69) and (70), respectively. We can obtain the condition for equivalent transformation of the two models.

Theorem 3. For any $\pi^{*} \in Z_{M U}, \pi^{*} \in Z_{A U}$.

Theorem 4. Assume $\pi^{*} \in Z_{A U}$. A necessary condition for $\pi^{*} \in$ $Z_{M U}$ is

$$
\lambda=2 E\left(Y_{T}\right)+2 E\left(W_{S, t_{T}}\right)
$$

The proofs of Theorems 3 and 4 are similar to those of Theorems 1 and 2 and are omitted.

Dynamic programming is employed to obtain the optimal solution of the following auxiliary model. The dynamic programming starts from the period $T-1$. The optimization problem is given as follows:

$$
\begin{aligned}
\max J_{T-1}\left(H_{T-1} \mid Y_{T-1}\right) \\
\quad=\max E\left\{-Y_{T}^{2}+\lambda Y_{T}-2 Y_{T} W_{S, t_{T}}\right\}
\end{aligned}
$$

$$
\begin{aligned}
&=\max E\{-\left(Y_{T-1} e_{C, T-1}+L_{T-1} H_{T-1}\right)^{2} \\
&\left.+\left(\lambda-2 W_{S, t_{T}}\right)\left(Y_{T-1} e_{C, T-1}+L_{T-1} H_{T-1}\right)\right\} \\
&=\max \{- H_{T-1}^{2} E\left(L_{T-1}^{2}\right) \\
&+ {\left[-2 Y_{T-1} E\left(e_{C, T-1} L_{T-1}\right)\right.} \\
&\left.+\lambda E\left(L_{T-1}\right)-2 E\left(W_{S, t_{T}} L_{T-1}\right)\right] H_{T-1} \\
&- Y_{T-1}^{2} E\left(e_{C, T-1}^{2}\right)+\lambda Y_{T-1} E\left(e_{C, T-1}\right) \\
&\left.-2 Y_{T-1} E\left(W_{S, t_{T}} e_{C, T-1}\right)\right\} .
\end{aligned}
$$

From (72), we have

$$
\begin{aligned}
& H_{T-1}^{*}=\frac{(\lambda / 2) E\left(L_{T-1}\right)-Y_{T-1} E\left(e_{C, T-1} L_{T-1}\right)-E\left(W_{S, t_{T}} L_{T-1}\right)}{E\left(L_{T-1}^{2}\right)}, \\
& J_{T-1}^{*}\left(Y_{T-1}\right)=-\omega_{T-1} Y_{T-1}^{2}+\left(\lambda \delta_{T-1}+K\right) Y_{T-1}+\frac{\lambda^{2}}{4} \beta_{T-1}+\gamma,
\end{aligned}
$$

where

$$
\begin{gathered}
\varphi_{T-1}=E\left(e_{C, T-1}^{2}\right)-\frac{E^{2}\left(e_{C, T-1} L_{T-1}\right)}{E\left(L_{T-1}^{2}\right)}, \\
\gamma_{T-1}=E\left(e_{C, T-1}\right)-\frac{E\left(L_{T-1}\right) E\left(e_{C, T-1} L_{T-1}\right)}{E\left(L_{T-1}^{2}\right)}, \\
K=2\left[\frac{E\left(W_{S, t_{T}} L_{T-1}\right) E\left(e_{C, T-1} L_{T-1}\right)}{E^{2}\left(L_{T-1}^{2}\right)}-E\left(W_{S, t_{T}} e_{C, T-1}\right)\right], \\
\gamma=\frac{\rho^{2}\left(L_{T-1}\right)}{E\left(L_{T-1}^{2}\right)}, \\
\left.E\left(W_{S, t_{T}} L_{T-1}\right)-\lambda E\left(L_{T-1}\right)\right] E\left(W_{S, t_{T}} L_{T-1}\right) \\
E\left(L_{T-1}^{2}\right)
\end{gathered}
$$

By analogy, we can derive the optimal $H_{i}(0 \leq i \leq T-2)$ 


$$
\begin{gathered}
H_{i}^{*}= \begin{cases}\frac{\left(\left(\lambda \delta_{T-1}+K\right) / 2 \omega_{T-1}\right) \prod_{k=i+1}^{T-2}\left(\delta_{k} / \omega_{k}\right) E\left(P_{i}\right)-M_{i} E\left(e_{C, i} P_{i}\right)}{E\left(P_{i}^{2}\right)}, & 0 \leq i \leq T-3, \\
\frac{\left(\left(\lambda \delta_{T-1}+K\right) / 2 \omega_{T-1}\right) E\left(P_{T-2}\right)-M_{T-2} E\left(e_{C, T-2} P_{T-2}\right)}{E\left(P_{T-2}^{2}\right)}, & i=T-2,\end{cases} \\
J_{i}^{*}\left(M_{i}\right)= \begin{cases}-M_{i}^{2} \prod_{k=i}^{T-1} \omega_{k}+\left(\lambda \delta_{T-1}+K\right) M_{i} \prod_{k=i}^{T-2} \delta_{k}+\frac{\left(\lambda \delta_{T-1}+K\right)^{2}}{4 \omega_{T-1}}\left[\sum_{k=i}^{T-3}\left(\beta_{k} \prod_{s=k+1}^{T-2} \frac{\delta_{s}^{2}}{\omega_{s}}\right)\right] & 0 \leq i \leq T-3, \\
+\frac{\left(\lambda \delta_{T-1}+K\right)^{2}}{4 \omega_{T-1}} \beta_{T-2}+\frac{\lambda^{2}}{4} \beta_{T-1}+\gamma, & i=T-2, \\
-\omega_{T-1} \omega_{T-2} M_{T-2}^{2}+\left(\lambda \delta_{T-1}+K\right) \delta_{T-2} M_{T-2}+\frac{\left(\lambda \delta_{T-1}+K\right)^{2}}{4 \omega_{T-1}} \beta_{T-2}+\frac{\lambda^{2}}{4} \beta_{T-1}+\gamma, & \end{cases}
\end{gathered}
$$

where

$$
\begin{gathered}
\varphi_{k}=E\left(e_{C, k}^{2}\right)-\frac{E^{2}\left(e_{C, k} L_{k}\right)}{E\left(L_{k}^{2}\right)}, \\
\gamma_{k}=E\left(e_{C, k}\right)-\frac{E\left(L_{k}\right) E\left(e_{C, k} L_{k}\right)}{E\left(L_{k}^{2}\right)},
\end{gathered}
$$$$
\rho_{k}=\frac{E^{2}\left(L_{k}\right)}{E\left(L_{k}^{2}\right)} .
$$

Substituting (73) and (75) into (64), the terminal capital of the firm can be expressed by

$$
Y_{i}= \begin{cases}Y_{0}\left[e_{C, 0}-\frac{L_{0} E\left(e_{C, 0} L_{0}\right)}{E\left(L_{0}^{2}\right)}\right]+\frac{\left(\left(\lambda \delta_{T-1}+K\right) / 2 \omega_{T-1}\right) \prod_{k=1}^{T-1}\left(\delta_{k} / 2 \omega_{k}\right) E\left(L_{0}\right)}{E\left(L_{0}^{2}\right)}, & i=1 ; \\ Y_{0} \prod_{k=0}^{i-1}\left[e_{C, k}-\frac{L_{k} E\left(e_{C, k} L_{k}\right)}{E\left(L_{k}^{2}\right)}\right]+\frac{\left(\lambda \delta_{T-1}+K\right)}{2 \omega_{T-1}} \sum_{g=0}^{i-2}\left\{\frac{L_{g} E\left(L_{g}\right)}{E\left(L_{g}^{2}\right)} \prod_{k=g+1}^{T-2} \frac{\delta_{k}}{\omega_{k}} \prod_{k=g+1}^{i-1}\left[e_{C, k}-\frac{L_{k} E\left(e_{C, k} L_{k}\right)}{E\left(L_{k}^{2}\right)}\right]\right\} & \\ +\frac{\left(\lambda \delta_{T-1}+K\right)}{2 \omega_{T-1}} \prod_{k=i}^{T-2} \frac{\delta_{k}}{\omega_{k}} \frac{L_{i-1} E\left(L_{i-1}\right)}{E\left(L_{i-1}^{2}\right)}, & 2 \leq i \leq T-2 ; \\ Y_{T-2}\left[e_{C, T-2}-\frac{L_{T-2} E\left(e_{C, T-2} L_{T-2}\right)}{E\left(L_{T-2}^{2}\right)}\right]+\frac{\lambda \delta_{T-1}+K}{2 \omega_{T-1}} \frac{L_{T-2} E\left(L_{T-2}\right)}{E\left(L_{T-2}^{2}\right)}, & i=T-1 ; \\ Y_{T-1}\left[e_{C, T-1}-\frac{L_{T-1} E\left(e_{C, T-1} L_{T-1}\right)}{E\left(L_{T-1}^{2}\right)}\right]+\frac{(\lambda / 2) L_{T-1} E\left(L_{T-1}\right)-L_{T-1} E\left(W_{S, t_{T}} L_{T-1}\right)}{E\left(L_{T-1}^{2}\right)} & i=T .\end{cases}
$$

Combining (77) with the necessary condition $\lambda=2 E\left(Y_{T}\right)+$ $2 E\left(W_{S, t_{T}}\right)$, we have

$$
\begin{aligned}
\lambda & =2 E\left(Y_{T}\right)+2 E\left(W_{S, t_{T}}\right) \\
& =2 \omega_{T-1}\left[M_{0} \prod_{k=0}^{T-1} \delta_{k}+E\left(W_{S, t_{T}}\right)\right]
\end{aligned}
$$

$$
\begin{aligned}
+K\left\{\sum_{g=0}^{T-4}\left\{\beta_{g} \prod_{k=g+1}^{T-2} \frac{\delta_{k}}{\omega_{k}} \prod_{k=g+1}^{T-1} \omega_{k}\right\}\right. \\
\left.+\frac{\delta_{T-2} \beta_{T-3}}{\omega_{T-2}} \prod_{k=T-2}^{T-1} \delta_{k}+\beta_{T-2} \delta_{T-1}\right\} .
\end{aligned}
$$


The solution of (78) is given by

$$
\lambda=\frac{2 \varphi_{T-1}\left[M_{0} \prod_{k=0}^{T-1} \gamma_{k}+E\left(W_{S, t_{T}}\right)\right]+K v}{\varphi_{T-1}\left(1-\rho_{T-1}\right)-\gamma_{T-1} v}
$$

where

$$
\begin{aligned}
v= & \sum_{g=0}^{T-4}\left\{\beta_{g} \prod_{k=g+1}^{T-2} \frac{\delta_{k}}{\omega_{k}} \prod_{k=g+1}^{T-1} \omega_{k}\right\} \\
& +\frac{\delta_{T-2} \beta_{T-3}}{\omega_{T-2}} \prod_{k=T-2}^{T-1} \delta_{k}+\beta_{T-2} \delta_{T-1} .
\end{aligned}
$$
(67) is

Therefore, the optimal solution of the proposed model

$$
H_{i}^{*}= \begin{cases}\frac{\left(\left(\lambda \delta_{T-1}+K\right) / 2 \omega_{T-1}\right) \prod_{k=i+1}^{T-2}\left(\delta_{k} / \omega_{k}\right) E\left(L_{i}\right)-Y_{i} E\left(e_{C, i} L_{i}\right)}{E\left(L_{i}^{2}\right)}, & 0 \leq i \leq T-2, \\ \frac{\left.\left(\lambda \delta_{T-1}+K\right) / 2 \omega_{T-1}\right) E\left(L_{T-2}\right)-Y_{T-2} E\left(e_{C, T-2} L_{T-2}\right)}{E\left(P_{T-2}^{2}\right)}, & i=T-1, \\ \frac{(\lambda / 2) E\left(L_{T-1}\right)-Y_{T-1} E\left(e_{C, T-1} L_{T-1}\right)-E\left(W_{S, t_{T}} L_{T-1}\right)}{E\left(L_{T-1}^{2}\right)}, & i=T-1,\end{cases}
$$

where $Y_{i}$ and $\lambda$ satisfy (77) and (79), respectively.

\section{Conclusion}

Stock index futures contracts provide an excellent tool for the risk management. However, the expiration date of the hedge is later than the delivery dates of all the index futures contracts that can be used. It is difficult for the hedger to directly use short index futures contracts for managing the long-term exposure in the stock market. The hedger has to choose the rollover hedge strategy. In this paper, we consider the problem of the rollover hedge strategy for the long-term exposure of a well-diversified portfolio. Two new models are presented to solve the problem. The proposed models are nonseparable in the sense of dynamic programming. Therefore, it is necessary to transform the proposed models into separable models. Using equivalent transformation technique, auxiliary models are constructed for the solution of the proposed models. Finally, the optimal numbers of index futures contracts have been derived by dynamic programming.

\section{Conflict of Interests}

The authors declare that they have no conflict of interests regarding the publication of this paper.

\section{Acknowledgments}

This research was supported by the Humanities and Social Science Youth Foundation of the Ministry of Education of China (no. 13YJC790028), Zhejiang Provincial Natural Science Foundation of China (Grant no. LQ13G010002), Research Project of Zhejiang Provincial Education Department (no. Y201328396), Major Project of the National Social
Science Foundation of China (no. 11\&ZD156), National Natural Science Foundation of China (Grant no. 71171176), and the Fundamental Research Funds for the Central Universities (SCUT no. 2014ZP0005).

\section{References}

[1] S. Figlewski, "Hedging performance and basis risk in stock index futures," Journal of Finance, vol. 39, no. 3, pp. 657-669, 1984.

[2] S. Figlewski, "Hedging with stock index futures: theory and application in a new market," The Journal of Futures Markets, vol. 5, no. 2, pp. 183-199, 1985.

[3] L. M. indahl, "Minimum variance hedge ratios for stock index futures: duration and expiration effects," The Journal of Futures Markets, vol. 12, no. 1, pp. 33-53, 1992.

[4] P. Holmes, "Stock index futures hedging: Hedge ratio estimation, duration effects, expiration effects and hedge ratio stability," Journal of Business Finance and Accounting, vol. 23, no. 1, pp. 63-77, 1996.

[5] H. C. Lee and C. Y. Chien, "Hedging performance and stock market liquidity: evidence from the taiwan futures market," Asia-Pacific Journal of Financial Studies, vol. 39, no. 3, pp. 396415, 2010.

[6] A. Ghosh, "Hedging with stock index futures: estimation and forecasting with error correction model," The Journal of Futures Markets, vol. 13, no. 7, pp. 743-752, 1993.

[7] B. Eftekhari, "Lower partial moment hedge ratios," Applied Financial Economics, vol. 8, no. 6, pp. 645-652, 1998.

[8] D. Lien and Y. K. Tse, "Hedging downside risk with futures contracts," Applied Financial Economics, vol. 10, no. 2, pp. 163170, 2000.

[9] S. S. Chen, C. F. Lee, and K. Shrestha, "On a mean-generalized semivariance approach to determining the hedge ratio," Journal of Futures Markets, vol. 21, no. 6, pp. 581-598, 2001.

[10] T. H. Park and L. N. Switzer, "Time-varying distributions and the optimal hedge ratios for stock index futures," Applied Financial Economics, vol. 5, no. 3, pp. 131-137, 1995. 
[11] G. Lypny and M. Powalla, "The hedging effectiveness of DAX futures," The European Journal of Finance, vol. 4, no. 4, pp. 345355, 1998.

[12] T. Choudhry, "Short-run deviations and optimal hedge ratio: evidence from stock futures," Journal of Multinational Financial Management, vol. 13, no. 2, pp. 171-192, 2003.

[13] T. Choudhry, "The hedging effectiveness of constant and timevarying hedge ratios using three Pacific Basin stock futures," International Review of Economics and Finance, vol. 13, no. 4, pp. 371-385, 2004.

[14] F. Pattarin and R. Ferretti, "The Mib30 index and futures relationship: econometric analysis and implications for hedging," Applied Financial Economics, vol. 14, no. 18, pp. 1281-1289, 2004.

[15] Y. Lai, C. W. S. Chen, and R. Gerlach, "Optimal dynamic hedging via copula-threshold-GARCH models," Mathematics and Computers in Simulation, vol. 79, no. 8, pp. 2609-2624, 2009.

[16] H. T. Lee, "Optimal futures hedging under jump switching dynamics," Journal of Empirical Finance, vol. 16, no. 3, pp. 446456, 2009.

[17] Y. Hou and S. Li, "Hedging performance of Chinese stock index futures: an empirical analysis using wavelet analysis and flexible bivariate GARCH approaches," Pacific-Basin Finance Journal, vol. 24, pp. 109-131, 2013.

[18] A. B. Sim and R. Zurbruegg, "Dynamic hedging effectiveness in South Korean index futures and the impact of the Asian financial crisis," Asia-Pacific Financial Markets, vol. 8, no. 3, pp. 237-258, 2001.

[19] J. Wang and H. Hsu, "Hedge ratio stability and hedging effectiveness of time-varying hedge ratios in volatile index futures markets: evidence from the Asian financial crisis," AsiaPacific Journal of Financial Studies, vol. 39, no. 5, pp. 659-686, 2010.

[20] W. Yang and D. E. Allen, "Multivariate GARCH hedge ratios and hedging effectiveness in Australian futures markets," Accounting and Finance, vol. 45, no. 2, pp. 301-321, 2005.

[21] C. Floros and D. V. Vougas, "Hedge ratios in Greek stock index futures market," Applied Financial Economics, vol. 14, no. 15, pp. 1125-1136, 2004.

[22] W. C. Pok, S. S. Poshakwale, and J. L. Ford, "Stock index futures hedging in the emerging Malaysian market," Global Finance Journal, vol. 20, no. 3, pp. 273-288, 2009.

[23] V. Aragó and E. Salvador, "Sudden changes in variance and time varying hedge ratios," European Journal of Operational Research, vol. 215, no. 2, pp. 393-403, 2011.

[24] A. Alizadeh and N. Nomikos, "A markov regime switching approach for hedging stock indices," Journal of Futures Markets, vol. 24, no. 7, pp. 649-674, 2004.

[25] J. Laws and J. Thompson, "Hedging effectiveness of stock index futures," European Journal of Operational Research, vol. 163, no. 1, pp. 177-191, 2005.

[26] P. Kofman and P. McGlenchy, "Structurally sound dynamic index futures hedging," Journal of Futures Markets, vol. 25, no. 12, pp. 1173-1202, 2005.

[27] Y. Wei, Y. Wang, and D. Huang, "A copula-multifractal volatility hedging model for CSI 300 index futures," Physica A: Statistical Mechanics and its Applications, vol. 390, no. 23-24, pp. 42604272, 2011.

[28] X. Wen, Y. Wei, and D. Huang, "Speculative market efficiency and hedging effectiveness of emerging chinese index futures market," Journal of Transnational Management, vol. 16, no. 4, pp. 252-269, 2011.
[29] E. Kostika and R. N. Markellos, "Optimal hedge ratio estimation and effectiveness using ARCD," Journal of Forecasting, vol. 32, no. 1, pp. 41-50, 2013.

[30] A. Neuberger, "Hedging long-term exposures with multiple short-term futures contracts," Review of Financial Studies, vol. 12, no. 3, pp. 429-459, 1999.

[31] J. E. Hilliard, "Analytics underlying the metallgesellschaft hedge: short term futures in a multi-period environment," Review of Quantitative Finance and Accounting, vol. 12, no. 3, pp. 195-219, 1999.

[32] D. Lien and D. R. Shaffer, "Multiperiod strip hedging of forward commitments," Review of Quantitative Finance and Accounting, vol. 18, no. 4, pp. 345-358, 2002.

[33] J. M. Godbey and J. E. Hilliard, "Adjusting stacked-hedge ratios for stochastic convenience yield: a minimum variance approach," Quantitative Finance, vol. 7, no. 3, pp. 289-300, 2007.

[34] G. Leobacher, "On a class of optimization problems emerging when hedging with short term futures contracts," Mathematical Methods of Operations Research, vol. 67, no. 1, pp. 65-90, 2008.

[35] Ó. Carchano and Á. Pardo, "Rolling over stock index futures contracts," Journal of Futures Markets, vol. 29, no. 7, pp. 684694, 2009.

[36] J. Fu, W. G. Zhang, Z. Yao, and X. Zhang, "Hedging the portfolio of raw materials and the commodity under the mark-to-market risk," Economic Modelling, vol. 29, no. 4, pp. 1070-1075, 2012.

[37] D. Li and W. L. Ng, "Optimal dynamic portfolio selection: multiperiod mean-variance formulation," Mathematical Finance, vol. 10, no. 3, pp. 387-406, 2000.

[38] R. W. Reid and S. J. Citron, "On noninferior performance index vectors," Journal of Optimization Theory and Applications, vol. 7, pp. 11-28, 1971. 


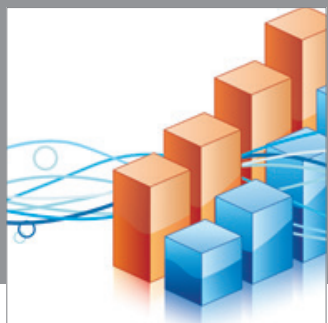

Advances in

Operations Research

mansans

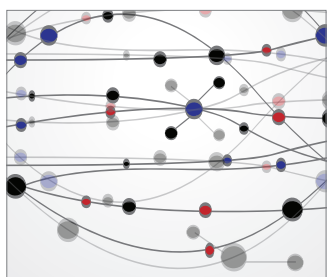

The Scientific World Journal
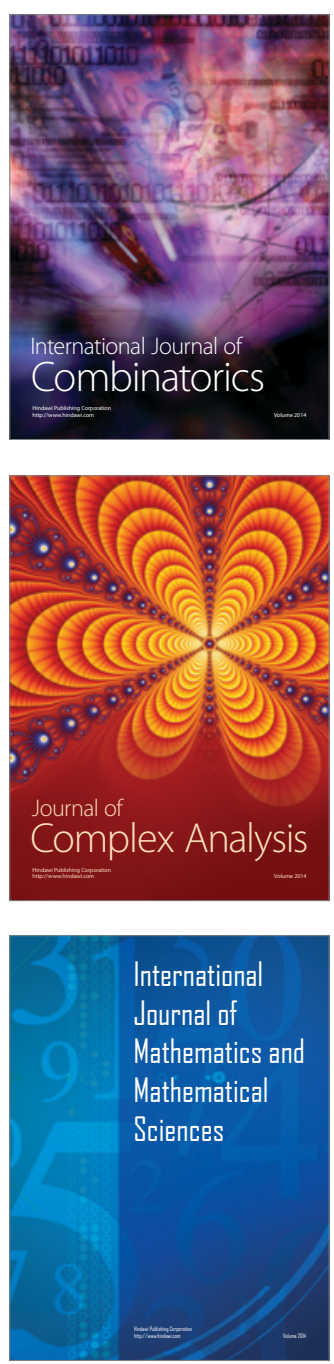
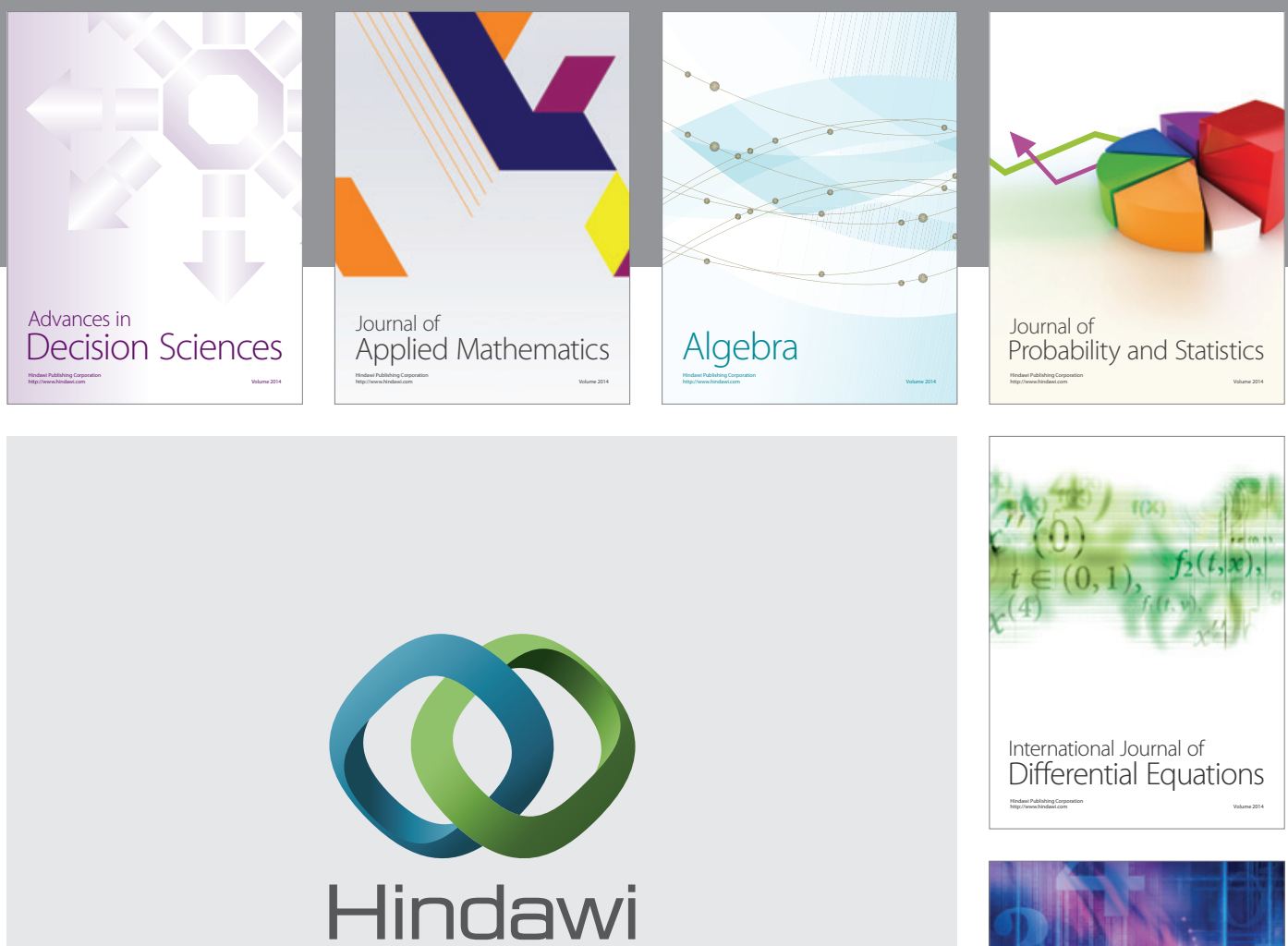

Submit your manuscripts at http://www.hindawi.com
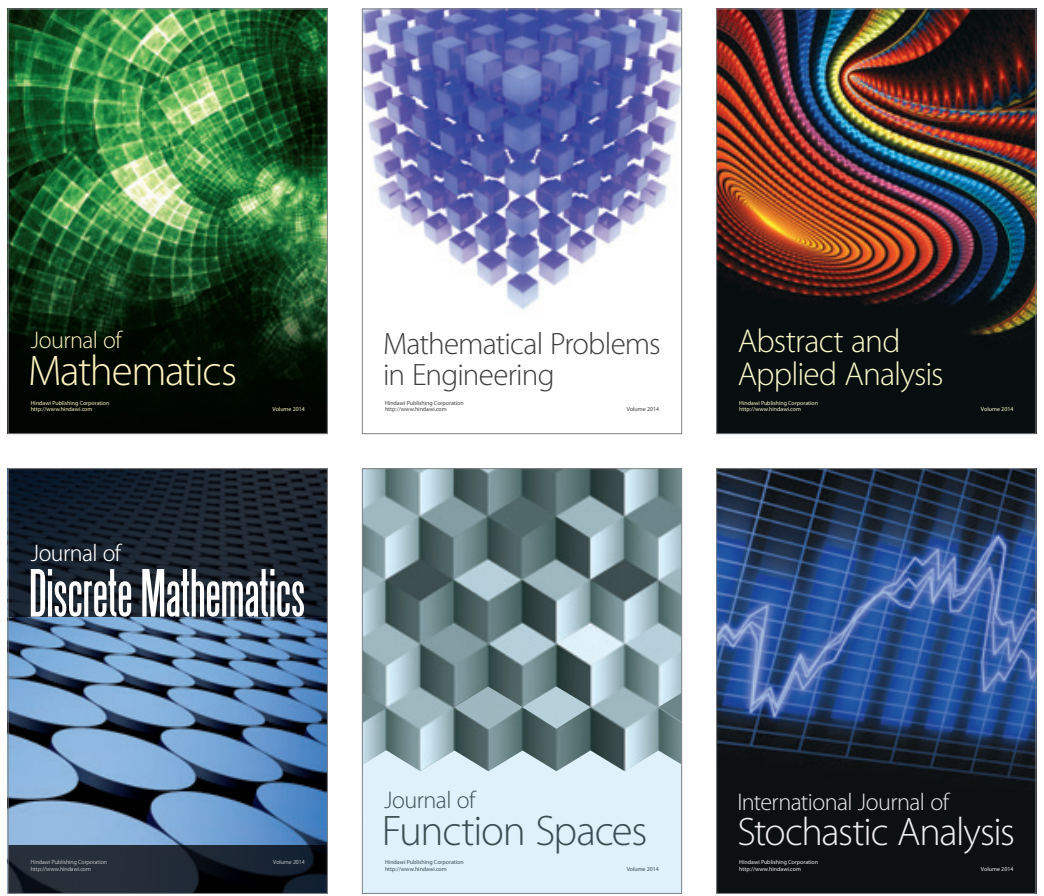

Journal of

Function Spaces

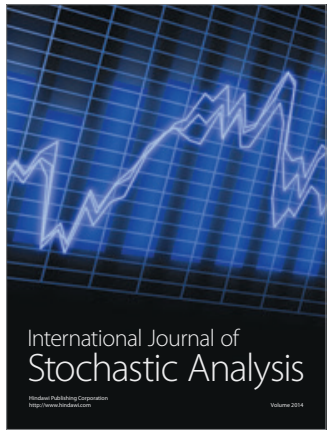

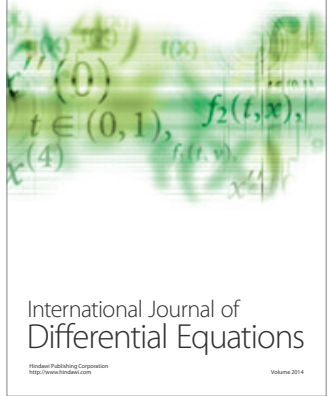
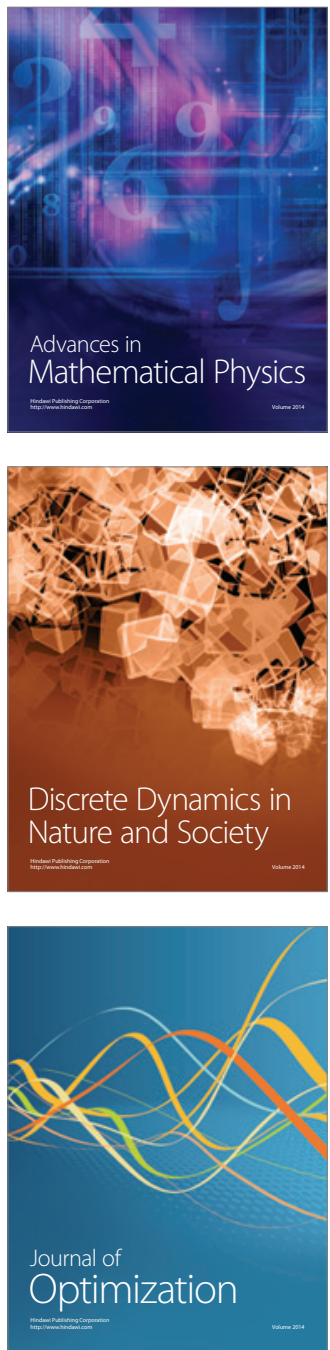